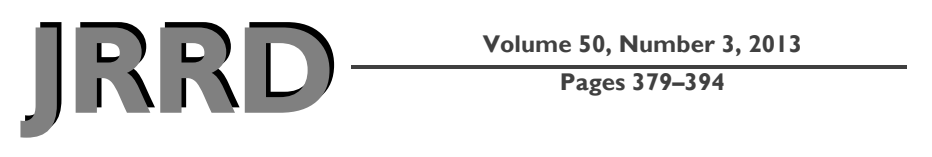

\title{
Estimating the patient's contribution during robot-assisted therapy
}

\author{
Marco Guidali, PhD $;^{{ }^{*}}$ Urs Keller, MSc; ${ }^{1}$ Verena Klamroth-Marganska, MD ${ }^{1}{ }^{\text {Tobias Nef, PhD; }}{ }^{2}$ Robert \\ Riener, PhD $^{1}$ \\ ${ }^{1}$ Sensory-Motor Systems Laboratory, ETH Zurich, Zurich, Switzerland; and Spinal Cord Injury Center, University Hos- \\ pital Balgrist, Zurich, Switzerland; ${ }^{2}$ Gerontechnology and Rehabilitation, ARTORG Center for Biomedical Engineer- \\ ing Research, University of Bern, Bern, Switzerland
}

\begin{abstract}
Robot-assisted therapy has become increasingly common in neurorehabilitation. Sophisticated controllers have been developed for robots to assist and cooperate with the patient. It is difficult for the patient to judge to what extent the robot contributes to the execution of a movement. Therefore, methods to comprehensively quantify the patient's contribution and provide feedback are of key importance. We developed a method comprehensively to estimate the patient's contribution by combining kinematic measures and the motor assistance applied. Inverse dynamic models of the robot and the passive human arm calculate the required torques to move the robot and the arm and build, together with the recorded motor torque, a metric (in percentage) that represents the patient's contribution to the movement. To evaluate the developed metric, 12 nondisabled subjects and 7 patients with neurological problems simulated instructed movement contributions. The results are compared with a common performance metric. The estimation shows very satisfying results for both groups, even though the arm model used was strongly simplified. Displaying this metric to patients during therapy can potentially motivate them to actively participate in the training.
\end{abstract}

Key words: cooperative control, dynamics, feedback, modeling, performance metric, rehabilitation, robot-aided therapy, robotics, stroke, upper limb.

\section{INTRODUCTION}

To promote effective neurorehabilitation, active participation of the patient is a key factor [1-3]. In conven- tional therapy, the therapist tries to motivate the patient with an attractive therapy concept. The therapist often touches the patient's limbs to assist him or her and estimates effort based on therapeutic experience. During robot-assisted therapy, the robot supports the patient's limb; the therapist is usually not in direct contact and, therefore, less able to assess effort. Many robots automatically adapt their support to the needs of the patient with a patient-cooperative control strategy [4-5]. The goal of these controllers is to assist as needed and ensure that the patient can perform the demanded movement. The disadvantage of these strategies is that the patient might find it convenient to rely too much on the robotic support, leading to decreased voluntary effort. Because the robot adapts its assistance levels to the needs of the patient, the therapist would not observe the decreased patient contribution to the movement. To account for this human lacking in the control loop, Wolbrecht et al. proposed a forgetting factor for the adaptation of assistance [6]. Another solution would be to put the patient in charge of

\footnotetext{
Abbreviations: $\mathrm{CAD}=$ computer-aided design, $\mathrm{DOF}=$ degree of freedom, FM = Fugl-Meyer, RMS = root-mean-square, $\mathrm{SD}=$ standard deviation.

*Address all correspondence to Marco Guidali, PhD; Sensory-Motor Systems Laboratory, ETH Zurich, Tannenstrasse 1, TAN E2, 8092 Zurich, Switzerland; 41446324270; fax: 41446327109. Email: marco.guidali@gmail.com http://dx.doi.org/10.1682/JRRD.2011.09.0172
} 
controlling his or her effort by providing feedback about movement contribution. To achieve this, an appropriate metric to measure the physical effort of the patient is required.

In task-oriented therapy, quantification of patient performance is difficult. One performance measure is the task itself, where time to finish or the number of achieved tasks can be measured and displayed as a score [7-8]. Many robots can also assess motor performance in terms of velocity, precision, or smoothness of trajectory [9-10]. Other studies used electromyography signals as indicators of effort [11]. In these applications, the score often depends only on the task performance and does not take the applied robotic assistance into account. Lünenburger et al. proposed a method for the gait rehabilitation robot Lokomat, where the interaction forces between patient and exoskeleton are measured and multiplied by a weighting function to obtain the biofeedback metric about the patient's participation during one gait cycle [12]. The arm robot RUPERT calculates the amount of assistance by dividing the work measured during a movement with a reference work value, obtained from a passive arm movement [13]. Ziherl et al. used reaching forces or mechanical work to evaluate subject performance [14]. Most methods depend on a measured reference value for a particular movement. This limits the application when many different movements are trained, e.g., activities of daily living training.

This article proposes and evaluates a method to measure a patient's contribution during robot-assisted training. With inverse dynamic models of the robot and the passive human limb, a patient's contribution to the movement is calculated based on his or her performance. To evaluate the proposed metric, we used the arm rehabilitation robot ARMin III. Twelve nondisabled subjects and seven patients with neurological problems simulated instructed contributions during robot-assisted arm movements.

\section{METHODS}

\section{Patient Contribution Estimation}

Many controllers in robot-assisted rehabilitation automatically adjust the amount of support to the point where the patient is able to perform the task. Therefore, the effective patient contribution would be important feedback for the patient and therapist. To calculate the torques contributed by the patient, the total torques acting on the robot and patient can be analyzed. The torques required to move the robot $\left(\tau_{r}\right)$ and the human arm $\left(\tau_{h}\right)$ have to be generated by the motors $\left(\tau_{\text {motor }}\right)$ and the user $\left(\tau_{\text {user }}\right)$ :

$$
\tau_{r}+\tau_{h}=\tau_{\text {user }}+\tau_{\text {motor }}
$$

Since many robots are not equipped with torque sensors in every single joint, $\tau_{\text {user }}$ can often not be measured directly. However, if the other torques of Equation (1) are known, $\tau_{\text {user }}$ can be calculated with-

$$
\tau_{\text {user }}=\tau_{r}+\tau_{h}-\tau_{\text {motor }}
$$

$\tau_{\text {motor }}$ can usually be measured or easily estimated [15]. The torques required to move the robot and the human arm could be assessed by passive calibration of a particular movement or dynamic models of the robot [16] and the human arm [17].

Direct feedback about $\tau_{\text {user }}$ might not be very comprehensible because the value strongly depends on the movement. Normalizing the $\tau_{\text {user }}$ with the $\tau_{h}$ required to perform an independent movement allows us to estimate the user's contribution $\left(C_{\text {user }}\right)$ in percentage of a full contribution:

$$
C_{u s e r}=100 \cdot \frac{\tau_{u s e r}}{\tau_{h}}=100 \cdot \frac{\tau_{r}+\tau_{h}-\tau_{\text {motor }}}{\tau_{h}}
$$

To make the calculation more robust against sensor noise and/or model inaccuracy, the $C_{u s e r}$ can be summed up over one movement, e.g., one reaching movement:

$$
C_{\text {user }}=100 \cdot \frac{\sum_{i=1}^{n} \tau_{\text {user }}}{\sum_{i=1}^{n} \tau_{h}}=100 \cdot \frac{\sum_{i=1}^{n}\left(\tau_{r}+\tau_{h}-\tau_{\text {motor }}\right)}{\sum_{i=1}^{n} \tau_{h}},
$$

where $n=$ number of samples captured during the movement. If the user is able to perform a movement without assistance, the motor torques equal the torques required to move the robot, which equals 100 percent for $C_{\text {user }}$. Moving a completely passive arm would result in 0 percent contribution. When the user acts against the movement direction, the movement contribution becomes negative. 
In the next sections, we explain the application of this method using the arm rehabilitation robot ARMin III. We decided to develop inverse dynamic models of the robot and the passive human arm to estimate the torques required to move these.

\section{Rehabilitation Robot}

We performed experiments with the arm rehabilitation robot ARMin III [18-19]. The device consists of 7 actuated degrees of freedom (DOFs) to assist a user during therapy (Figure 1). The exoskeleton is attached to the user's arm with one cuff on the upper arm and one on the lower arm. Redundant position sensors are integrated in each joint. A rope connects a spring to counterbalance the weight of the exoskeleton with the upper arm. A torque sensor is mounted at the output flange of axis 2 .

\section{Inverse Dynamic Robot Model}

The ARMin III was modeled as a multibody system with 5 DOF. We neglected axes 6 and 7, wrist and hand movement, because they have little influence on the dynamics due to their relatively low mass $(4.58 \%$ and $1.53 \%$ of total mass, respectively) and small ranges of motion. We added the masses of axes 6 and 7 to body 5 . We derived the equations of motion for the dynamic model of the ARMin III by using the method of Lagrange (second kind) with-

$$
\frac{d}{d t}\left(\frac{\partial T}{\partial \dot{\boldsymbol{q}}}\right)-\frac{\partial T}{\partial \boldsymbol{q}}+\frac{\partial V}{\partial \boldsymbol{q}}=\boldsymbol{\tau}_{r}
$$

where $T=$ kinetic energy and $V=$ potential energy. Rearranged and sorted, the equations of motion can be written in a compact matrix form for the joint angles $q=\left(q_{1}, q_{2}\right.$, $\left.q_{3}, q_{4}, q_{5}\right)^{\tau}$ as-

$$
\boldsymbol{\tau}_{r}=\mathbf{M}_{r}(\boldsymbol{q}) \ddot{\boldsymbol{q}}+\boldsymbol{\eta}_{r}(\boldsymbol{q}, \dot{\boldsymbol{q}})+\boldsymbol{\eta}_{e}(\boldsymbol{q}, \dot{\boldsymbol{q}})
$$

$M_{r}(q)$ describes the mass matrix containing the moments of inertia from the bodies and its actuators. Gravitational, damping, and Coriolis torques are subsumed in $\boldsymbol{\eta}_{r}(\boldsymbol{q}, \dot{\boldsymbol{q}})$. The term $\boldsymbol{\eta}_{e}(\boldsymbol{q}, \dot{\boldsymbol{q}})$ describes all other effects (e.g., friction of the actuators). For our robot, we decided to neglect Coriolis torques and damping torques due to the relatively slow movements used in arm therapy and their small influence on the dynamics of a robot [20-21].

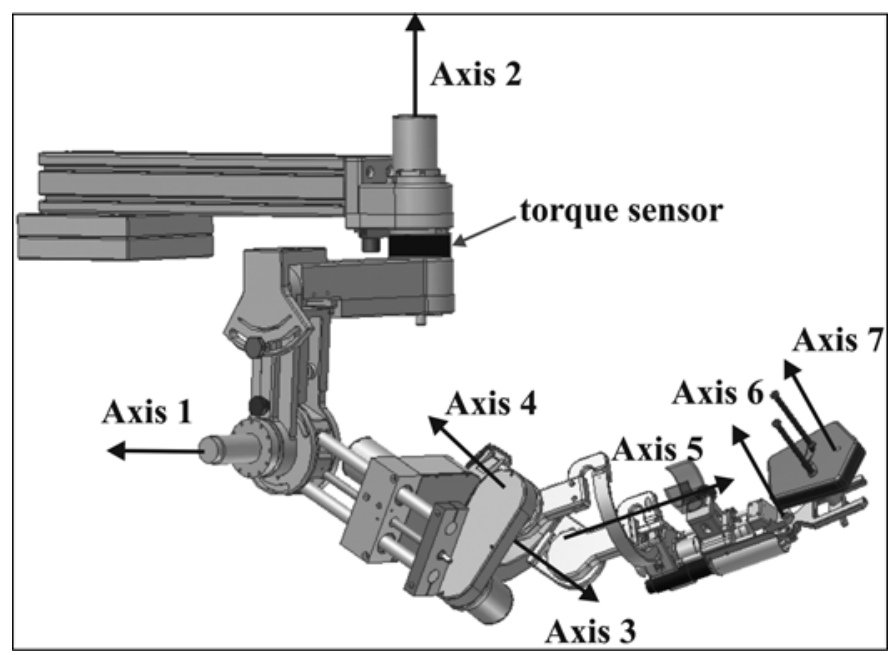

Figure 1.

Schematic of ARMin III with 7 degrees of freedom (axes 1-7) and torque sensor.

Therefore, we get a simplified equation of motion out of Equation (6):

$$
\boldsymbol{\tau}_{r}=\mathbf{M}_{r}(\boldsymbol{q}) \ddot{\boldsymbol{q}}+\mathbf{G}_{r}(\boldsymbol{q})+\boldsymbol{\eta}_{e}(\boldsymbol{q}, \dot{\boldsymbol{q}})
$$

where $G_{r}(q)=$ the gravitation of the exoskeleton.

We obtained the mass of the five bodies by measuring the disassembled parts with a scale [18]. The centers of mass have been calculated by using a computer-aided design (CAD) model of the ARMin III and the data sheets of the motors and gears. We used a symbolic math software program (Mathematica, Wolfram; Champaign, Illinois) to solve the equations of motion to obtain $M_{r}(q)$ and $G_{r}(q)$.

Additional effects acting on the system are described by $\boldsymbol{\eta}_{e}(\boldsymbol{q}, \dot{\boldsymbol{q}})$. We identified and modeled friction in the gears and the effect of the spring that counterbalances the robotic upper arm. During the identification process of these, we observed a high influence of the bending electronic cables. This influence on the system could also be modeled and identified, so the additional effects can be described by-

$$
\boldsymbol{\eta}_{e}(\boldsymbol{q}, \dot{\boldsymbol{q}})=\boldsymbol{\tau}_{f}+\boldsymbol{\tau}_{s}+\boldsymbol{\tau}_{c}
$$

where $\tau_{f}=$ torque induced by friction, $\tau_{s}=$ torque produced by the counterbalance spring, and $\tau_{c}=$ torque generated by the electronic cables. 


\section{Friction Model}

Friction of the motor gear combination is velocity dependent and identified by using a constant velocity controller [22]. We moved each joint with constant velocities from 0.1 to $60.0^{\circ} / \mathrm{s}$ over its entire range of motion. We recorded the required motor torque to maintain a constant velocity during the movement. We used this data to fit a common friction model that can be described by-

$$
F_{f}(v)=F_{f c}+\left(F_{f s}-F_{f c}\right) \cdot e^{-v v_{s}}+k_{f v} \cdot v,
$$

where $F_{f s}=$ static friction, $F_{f c}=$ Coulomb friction, $v_{s}=$ Stribeck velocity, and $k_{f v} \bullet v=$ viscous friction [23].

In the range of $F_{f s}$, the correct torque cannot be measured with the position sensors of the ARMin III. Therefore, we reduced the model to the Coulomb and viscous part. In our case, friction induces a torque, so Equation (9) can be reformulated to-

$$
\tau_{f_{i}}\left(\dot{q}_{i}\right)=\tau_{f c_{i}} \cdot\left(1-e^{-\dot{q}_{i} \dot{q}_{s_{i}}}\right)+k_{f v_{i}} \cdot \dot{q}_{i}
$$

where $i=$ axis number. Figure 2 shows the mean torque required to maintain the velocities and the identified model for axis 4 .

\section{Cable Model}

During the identification process of the motor gear friction, we observed a strong influence of the electronic cables attached to the exoskeleton. The effect is distinctive, particularly between the first two joints where the cable harness consists of 14 electronic cables. Analyzing the disturbance of the cables on the friction measurement showed a strong relationship between joint position and disturbance torque in the affected axis. Therefore, we modeled the torque $\left(\tau_{C i}\right)$ induced by the bending of the cable in axis $i$ as a spring acting on the system:

$$
\tau_{c_{i}}\left(q_{i}\right)=k_{c_{i}} \cdot\left(q_{i}-q_{c_{i}}\right),
$$

where $k_{C i}=$ the spring stiffness in $\frac{N m}{r a d}$ and $q_{C i}=$ the equilibrium joint position where the disturbance torque is zero.

\section{Counterbalance Spring Model}

One of the safety features of the ARMin III is the spring that counterbalances the orthosis when the motors are off. The torque applied by the spring acts only on the arm elevation axis and can, therefore, be described as a function of the corresponding joint angle $q_{1}$. Preliminary tests showed that a second-order polynomial would be appropriate to model this effect:

$$
\tau_{s}\left(q_{1}\right)=c_{2} q_{1}^{2}+c_{1} q_{1}+c_{0} \quad .
$$

We identified the coefficients $c_{0}, c_{1}$, and $c_{2}$ of the model by measuring the required torque for holding different arm elevation positions with the motors. Subtracting the gravity of the robot, the remaining torque is the one induced by the spring. We fit the obtained data with the polynomial of Equation (12). Since the spring only has an influence on axis 1 , the total spring torque vector is $\tau_{s}(q)=\left[\tau_{s}\left(q_{1}\right), 0,0,0,0\right]^{\tau}$.

Summing all modeled effects, we can describe the inverse dynamic model of the ARMin III with this equation:

$$
\boldsymbol{\tau}_{r}=\boldsymbol{M}_{r}(\boldsymbol{q}) \ddot{\boldsymbol{q}}+\boldsymbol{G}_{r}(\boldsymbol{q})+\boldsymbol{\tau}_{f}(\dot{\boldsymbol{q}})+\boldsymbol{\tau}_{c}(\boldsymbol{q})+\boldsymbol{\tau}_{s}(q) .
$$

\section{Inverse Dynamic Passive Human Arm Model}

The dynamics of the human arm are very complex and not all effects are yet fully understood. Since for our application the model should be easily identified and does not have to capture all effects, we decided to model the human arm as a passive multibody system. We approximated the mass of the upper arm, the lower arm, and the hand as point masses. We used anthropometric data from Winter to derive this arm model for different arm sizes and weights [24]. The final model can be adjusted with the length of the upper and lower arm, the length of the hand, and the body weight of the subject, all of which can be easily measured. The output of the model is the torque $\left(\tau_{h}\right)$ required to move a passive human arm at position $(q)$ with velocity $(\dot{\boldsymbol{q}})$ and acceleration $(\ddot{q})$ :

$$
\boldsymbol{\tau}_{h}=\boldsymbol{M}_{h}(\boldsymbol{q}) \ddot{\boldsymbol{q}}+\boldsymbol{G}_{h}(\boldsymbol{q})+\boldsymbol{S}_{h}(\boldsymbol{q}, \dot{\boldsymbol{q}}, \ddot{\boldsymbol{q}})
$$

where $M_{h}=$ the mass matrix of the human arm and $G_{h}=$ the gravitational torques derived from the Winter data. The function $S_{h}$ defines the dynamics of the arm that cannot be captured with inertia or gravity. Possible effects are arm stiffness, damping, or muscle tone [25]. Measurements of the passive human arm showed that for the tested movements, the rest dynamics seem to mostly depend on the joint angle. Since the purpose of this 
model is to roughly estimate the required torque for a passive arm movement, we decided to simplify the function $S_{h}$ for our first approach to this position-dependency:

$$
\mathbf{S}_{h}(\boldsymbol{q})=\boldsymbol{K}_{s} \cdot\left(\boldsymbol{q}-\boldsymbol{q}_{0}\right),
$$

where $K_{s}=$ a diagonal matrix denoting the linear relationship between joint angle $\left(q_{i}\right)$ and torque at joint $i$. The vector $\left(q_{0}\right)$ is the equilibrium joint position where $S_{h}$ is zero.

A clear limitation of this simplified model is that it is not capable of capturing all impairment characteristics of persons with stroke. For example, pathological synergies or velocity-dependent joint stiffness and damping cannot be explained.

\section{Experimental Evaluation}

We divided the evaluation into two steps. First, we validated the inverse dynamic model of the ARMin III using a position controller to follow different trajectories with the robot. During this experiment, no user was inside the robot. We measured the required motor torque to move the robot and compared it with the inverse dynamic model of the ARMin III. We used two common benchmarks [26], the root-mean-square (RMS) error $\left(E_{R M S}\right)$ and the coefficient of determination $\left(R^{2}\right)$, to rate the developed model:

$$
\begin{gathered}
E_{R M S}=\sqrt{\frac{1}{n} \sum_{i=1}^{n}\left(\tau_{\text {motor }}(i)-\tau_{r}(i)\right)^{2}} \text { and } \\
R^{2}=1-\frac{\sum_{i=1}^{n}\left(\tau_{\text {motor }}(i)-\tau_{r}(i)\right)^{2}}{\sum^{n}\left(\tau_{\text {motor }}(i)-\bar{\tau}_{\text {motor }}\right)^{2}}
\end{gathered}
$$

where $n=$ the number of samples and $\bar{\tau}_{\text {motor }}=$ the mean motor torque during the measured range.

In the second step, we evaluated the inverse dynamic human arm model together with the user contribution estimation. We chose two movements to validate the method. We chose a horizontal shoulder movement with axis 2 , because there are no gravity effects in this movement and because the torque sensor in this axis gives an additional reference. The second movement was an arm elevation with axis 1 , because this is usually quite difficult for users. We compared our model-based approach to estimate $C_{\text {user }}$ with a method where the contribution is calculated with a measured reference torque and the support torque. The support torque $\left(\tau_{\text {support }}\right)$ is the difference between applied motor torque and torque required to move the robot:

$$
\tau_{\text {support }}=\tau_{\text {motor }}-\tau_{r}
$$

The reference or calibration torque ( $\left.\tau_{\text {support,ref }}\right)$ can be obtained from a passive movement. Similar to the proposed method, $C_{\text {user }}$ can be calculated for one movement:

$$
C_{\text {user }}^{\prime}=100 \cdot\left(1-\frac{\frac{1}{n} \sum_{i=1}^{n} \tau_{\text {support }}}{\bar{\tau}_{\text {support,ref }}}\right)=100 \cdot\left(1-\frac{\frac{1}{n} \sum_{i=1}^{n}\left(\tau_{\text {motor }}-\tau_{r}\right)}{\left(\bar{\tau}_{\text {motor }, \text { ef }}-\bar{\tau}_{r, \text { ref }}\right)}\right) \text {, }
$$

where $\bar{\tau}_{\text {motor, ref }}$ and $\bar{\tau}_{r, \text { ref }}=$ the mean torques obtained from a measurement with a passive human arm.

Beside the evaluation with human subjects, we developed a mock-up model of the human arm. The mock-up model consists of two pipes representing the upper and lower arm, together with the hand. The upperarm pipe has a diameter of $100 \mathrm{~mm}$, a length of $320 \mathrm{~mm}$, and a weight of $1.8 \mathrm{~kg}$. The lower-arm/hand pipe has a diameter of $90 \mathrm{~mm}$, a length of $300 \mathrm{~mm}$, and a weight of $1.7 \mathrm{~kg}$. We used this mock-up to validate the gravity and inertia terms of the passive human arm model.

\section{Protocol}

To validate the user's contribution estimation, we recruited 12 nondisabled subjects and 7 patients with neurological problems. The task was to perform two different single-joint movements with the ARMin III. The first movement was an arm elevation task, requiring the participant to lift his or her arm from $45^{\circ}$ to $110^{\circ}$ (axis 1) with the arm pointing forward and the elbow fully extended. The second movement was a horizontal shoulder movement from $0^{\circ}$ to $90^{\circ}$ (axis 2) with a fixed-arm elevation angle of $60^{\circ}$ and a fully extended elbow. We calculated reference trajectories by using the minimal angular jerk method and a mean desired velocity of $20 \%$ s.

We instructed the nondisabled subjects to simulate four different conditions of participation: "passive," "active," "resistive," and "half active." The patients with neurological problems completed only the passive, active, and resistive conditions; we did not instruct them to perform the half active movement. Participants performed each movement three times. Simple visual feedback showed the current and desired joint angles, as well 
as the start and target positions, using small circles on a computer monitor. The nondisabled subjects (S1-S12) were, on average, 33.5 years old (range: $27-52 \mathrm{yr}$ ); five were male and seven were female. Table 1 lists the characteristics of the patients with neurological problems (P1-P7). Except for one, all were patients severely to moderately affected by chronic stroke with recently assessed Fugl-Meyer (FM) scores. P1 had Guillain-Barré Syndrome [27] and did not have a clinical FM score.

During the passive condition, we instructed the participants to relax and let the robot move the arm. In this mode, a position controller guided the arm along the reference trajectory. This condition should correspond to 0 percent subject contribution to the movement; therefore, we also used this to identify the individual passive arm model parameters for each participant.

In the active condition, we instructed the participants to actively perform the movement. This condition should correspond to 100 percent contribution for nondisabled subjects. The ARMin III itself was compensated for using the gravity, friction, spring, and cable model of the device. Nondisabled subjects received no further support from the controller and were completely free in timing the movement. The patients with neurological problems were also instructed to contribute as much as possible but received support from a unidirectional position controller because most of them were unable to perform the movements without any assistance. The assistance was impedance-based and only active when the patient was behind the reference position. We used the deviation from the reference trajectory and its speed profile to adapt the support.

The instruction for the resistive condition was for the participant to oppose the movement. We used the same position controller as in the passive condition. Resisting the movement should result in a negative percent contri- bution to the movement. Since the ARMin III was not designed to apply high forces to the arm, a strong participant could stop it. Therefore, we instructed all participants to limit their resistance so that the movement could still be completed by the position controller.

Only the nondisabled subjects completed the fourth condition, half active. Here, nondisabled subjects had to participate during the movement with limited effort to simulate a weak user. We instructed them to try to reach a movement contribution of 50 percent. A unidirectional position controller provided assistance. This allowed them to move ahead of the reference trajectory. If they were slower than the reference, they were supported by the ARMin III.

\section{Data Analysis}

For each participant, we identified the passive arm model with the passive movements. Assuming that the model of the ARMin III and the torque measurement are exact and the user is completely passive, the torque required to move the passive arm is the difference between the two:

$$
\boldsymbol{\tau}_{h}=\boldsymbol{\tau}_{\text {motor }}-\boldsymbol{\tau}_{r}=\boldsymbol{M}_{h}(\boldsymbol{q}) \ddot{\boldsymbol{q}}+\boldsymbol{G}_{h}(\boldsymbol{q})+\mathbf{S}_{h}(\boldsymbol{q})
$$

The mass matrix $\left(M_{h}\right)$ and the gravity matrix $\left(G_{h}\right)$ are already defined for the participants using their body weight and height and the length of their arm segments. Solving Equation (20) for the unknown part $\left(S_{h}\right)$ of the model leads to-

$$
\boldsymbol{S}_{h}(q)=\boldsymbol{\tau}_{\text {motor }}-\boldsymbol{\tau}_{r}-\boldsymbol{M}_{h}(\boldsymbol{q}) \ddot{\boldsymbol{q}}-\boldsymbol{G}_{h}(\boldsymbol{q})=\boldsymbol{k}_{s} \cdot\left(\boldsymbol{q}-\boldsymbol{q}_{0}\right) \cdot
$$

Table 1.

\begin{tabular}{|c|c|c|c|c|c|}
\hline Patient & Incident & Sex & Age (yr) & $\begin{array}{c}\text { Time Since } \\
\text { Incident (yr) }\end{array}$ & $\begin{array}{c}\text { FM } \\
\text { (score) }\end{array}$ \\
\hline 1 & GBS & $\mathrm{M}$ & 59 & 1 & - \\
\hline 2 & Stroke & M & 47 & 2 & $40(23)$ \\
\hline 4 & Stroke & M & 66 & 4.5 & $18(16)$ \\
\hline 5 & Stroke & M & 38 & 1.5 & $32(26)$ \\
\hline
\end{tabular}

Characteristics of patients with neurological problems. 
With linear least-squares method optimization, we identified the parameters $k_{s i}$ and $q_{0 i}$ for the two measured joints $i$. We took the mean values over the three passive movements as individual model parameters.

\section{RESULTS}

\section{Inverse Dynamic Robot Model}

We evaluated the inverse dynamic model of the ARMin III with measurements from position-controlled movements with no user in the device. We recorded kinematic data $(q)$ and the applied motor torque $\left(\tau_{\text {motor }}\right)$ during multijoint movements.

We used this data to calculate the RMS error $\left(E_{R M S}\right)$ and the coefficient of determination $\left(R^{2}\right)$ according to Equations (16) and (17). Table 2 lists the results for the five modeled axes.

Table 3 summarizes the identified friction and cable coefficients. Axis 1 was not influenced by any moving cable. The attachment points of the cables are fixed to the exoskeleton. Therefore, the cable length and stiffness do not change over time, assuming that material fatigue of the cable is negligible. Figure 3 shows the influence of the cables on a movement with constant velocity.

Table 2.

Root-mean-square error $\left(E_{R M S}\right)$ and coefficient of determination $\left(R^{2}\right)$ of measured torque and model prediction.

\begin{tabular}{ccc}
\hline Axis & $\boldsymbol{R}^{\mathbf{2}}$ & $\boldsymbol{E}_{\boldsymbol{R M S}}(\mathbf{N m})$ \\
\hline 1 & 0.870 & 3.38 \\
2 & 0.962 & 0.62 \\
3 & 0.912 & 0.67 \\
4 & 0.874 & 1.25 \\
5 & 0.815 & 0.81 \\
\hline \hline
\end{tabular}

Table 3.

Identified friction and cable model parameters of ARMin III.

\begin{tabular}{cccccc}
\hline \multirow{2}{*}{ Axis } & \multicolumn{2}{c}{ Cable Parameter } & & \multicolumn{2}{c}{ Friction Parameter } \\
\cline { 2 - 3 } \cline { 5 - 6 } & $\begin{array}{c}\boldsymbol{k}_{\boldsymbol{C}} \\
\left(\mathbf{N m} /{ }^{\circ}\right)\end{array}$ & $\begin{array}{c}\boldsymbol{q}_{\boldsymbol{C}} \\
\left({ }^{\circ}\right)\end{array}$ & & $\begin{array}{c}\boldsymbol{T}_{\boldsymbol{f C}} \\
\mathbf{( N m )}\end{array}$ & $\begin{array}{c}\boldsymbol{k}_{\boldsymbol{f}} \\
\left(\mathbf{N m s} /{ }^{\circ}\right)\end{array}$ \\
\hline 1 & 0.0 & 0.0 & & 8.22 & 0.132 \\
2 & 0.016 & 41.0 & & 1.17 & 0.027 \\
3 & 0.015 & 10.05 & & 5.90 & 0.0065 \\
4 & 0.014 & 0.0 & & 1.07 & 0.013 \\
5 & 0.0078 & 54.5 & & 0.28 & 0.0 \\
\hline \hline
\end{tabular}

The Appendix (available online only) shows example trajectories of the model and the measured torque (Figure 2 in Appendix, available online only).

\section{Inverse Dynamic Passive Human Arm Model}

We used a mock-up model of the human arm to validate the developed arm model for joints 1 (arm elevation) and 2 (horizontal shoulder rotation). We measured the exact properties of the mock-up and used them in the inverse dynamic arm model to calculate gravity and inertia effects. With an arm-elevation movement, we validated the quality of the gravity component by moving with a constant speed of $20^{\circ} / \mathrm{s}$. We used the horizontal shoulder movement to validate the inertia component of the model. Figure 4 shows that the dynamic passive arm model is able to reproduce the measured torques required to move the mock-up.

\section{Patient Contribution Estimation}

First, we used the passive movements of the experiment to optimize the inverse dynamic passive human arm model. We also used them to identify the mean support torque required to perform that particular movement. We could identify the model parameters for the arm-elevation axis for all participants. For the horizontal shoulder movement, we could not identify the passive human arm

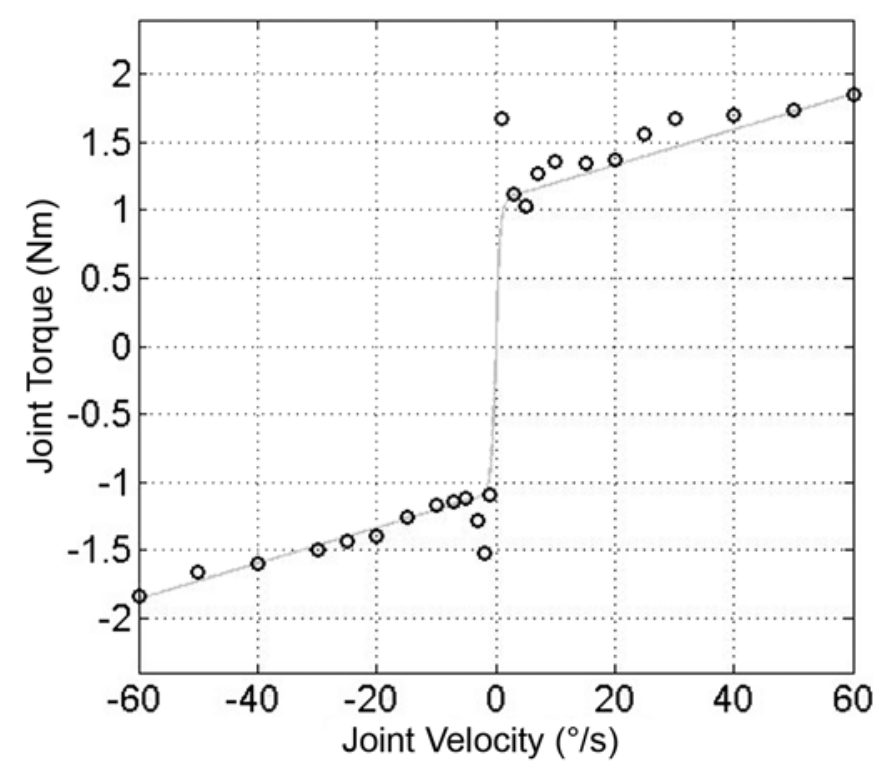

Figure 2.

Identified friction in axis 4 (elbow flexion and extension) are represented by black circles. Derived model is displayed as gray line. 
JRRD, Volume 50, Number 3, 2013

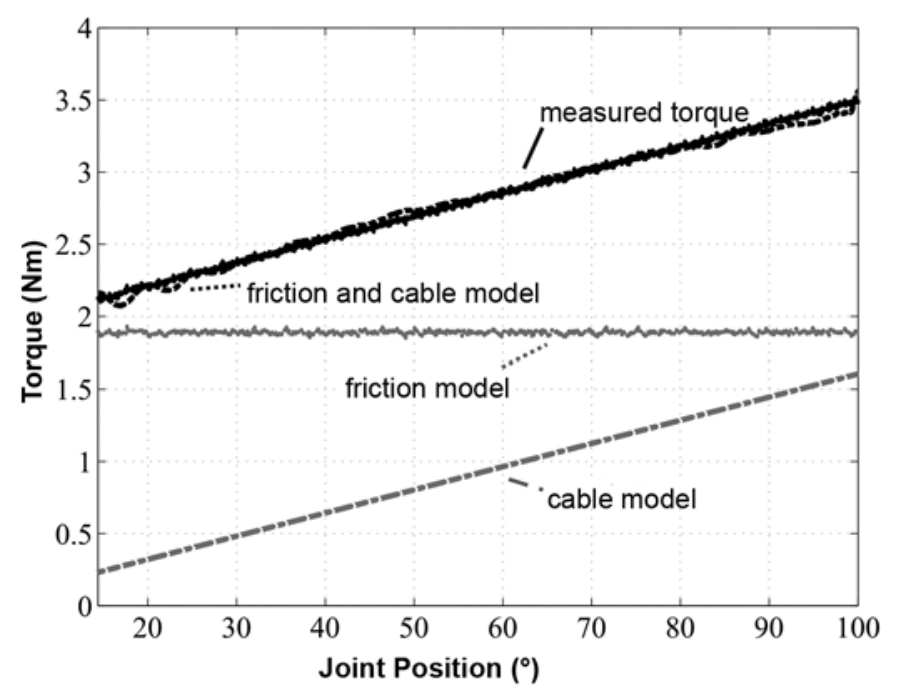

Figure 3.

Required torque to move axis 2 (horizontal shoulder rotation) with constant velocity of $15 \%$ and friction and cable model to predict this torque with position and velocity information.

model for one patient with a neurological problem and one nondisabled subject due to high variations in the recorded motor torques to move the arm. Table 4 lists the identified passive human arm model parameters for joints
1 (arm elevation) and 2 (horizontal shoulder rotation) according to Equation (15) for all participants.

Note that moving a passive patient's arm required more support from the ARMin III than moving a passive nondisabled subject's arm. This is also reflected by the higher arm model parameters of patients with neurological problems. Figure 5 shows examples of the calculated human arm model torque and the measured torque for two nondisabled subjects and two patients with neurological problems. We also recorded data from the torque sensors at axis 2 during the movement and compared them with the model.

We later used the identified individual dynamic passive arm model to calculate the user contribution $\left(C_{\text {user }}\right)$ for the instructed condition. We used the measured reference support torque to calculate $C^{\prime}{ }_{\text {user }}$ (Equation (19)).

\section{Arm Elevation Movement}

For the nondisabled subjects, we calculated a mean contribution of $1.94 \pm 1.72$ percent (mean \pm standard deviation [SD]) for the passive arm elevation movements. During the active condition, the estimated contribution was, on average, $102.90 \pm 7.38$ percent. For the half active condition, we calculated a mean $C_{\text {user }}$ of $57.40 \pm 17.15$ percent. In trials where the nondisabled

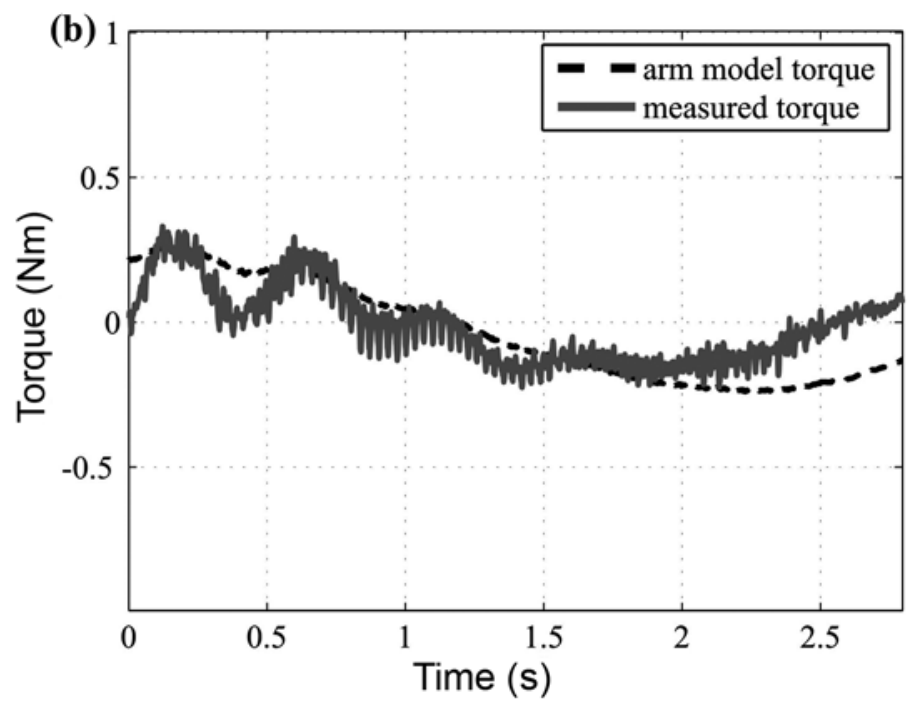

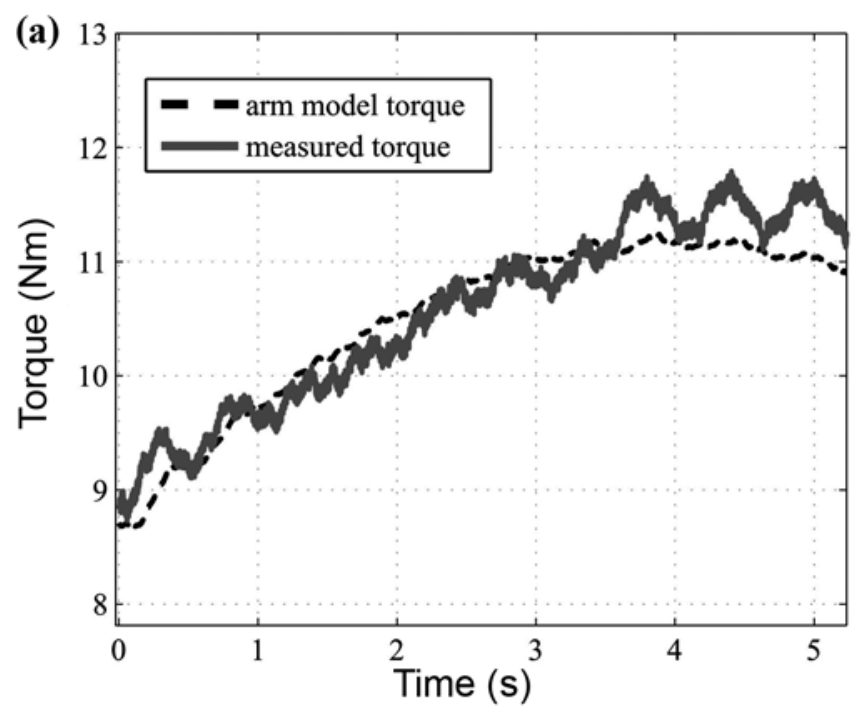

Figure 4.

Experiments with mock-up model of human arm. Mock-up consisted of two pipes. Upper-arm pipe has diameter of $100 \mathrm{~mm}$, length of $320 \mathrm{~mm}$, and weight of $1.8 \mathrm{~kg}$. Lower-arm/hand pipe has diameter of $90 \mathrm{~mm}$, length of $300 \mathrm{~mm}$, and weight of $1.7 \mathrm{~kg}$. (a) Torque trajectories for position-controlled arm elevation movement with constant speed. (b) Inertia effects for position-controlled horizontal shoulder movement with minimal jerk profile. 
Table 4.

Identified passive human arm parameters.

\begin{tabular}{|c|c|c|c|c|}
\hline Participant & $k_{s 1}(\mathrm{Nm} / \mathrm{rad})$ & $q_{01}(\mathrm{rad})$ & $k_{s 2}(\mathrm{Nm} / \mathrm{rad})$ & $q_{02}(\mathrm{rad})$ \\
\hline S1 & 6.60 & 1.33 & 1.68 & 0.44 \\
\hline $\mathrm{S} 2$ & 5.00 & 1.00 & 1.17 & 0.11 \\
\hline $\mathrm{S} 4$ & 3.99 & 1.00 & - & - \\
\hline S5 & 1.47 & 1.05 & 0.44 & -2.50 \\
\hline S6 & 3.06 & 1.42 & 0.76 & 0.026 \\
\hline S8 & 4.02 & 0.87 & 2.08 & 0.09 \\
\hline S9 & 6.80 & 0.96 & 1.03 & 0.24 \\
\hline $\mathrm{S} 10$ & 3.75 & 1.31 & 0.80 & -0.21 \\
\hline S11 & 4.40 & 1.14 & 1.04 & 0.29 \\
\hline S12 & 8.43 & 1.19 & 0.45 & -0.78 \\
\hline P3 & 10.37 & 0.65 & 1.75 & -1.77 \\
\hline P4 & 9.43 & 1.06 & 2.05 & -0.10 \\
\hline P5 & 11.84 & 0.68 & 0.88 & -1.43 \\
\hline P6 & 9.70 & 1.42 & 7.70 & 0.10 \\
\hline P7 & 5.85 & 0.79 & 0.003 & 0.64 \\
\hline Mean $\pm \mathrm{SD}$ & $10.36 \pm 2.40$ & $0.84 \pm 0.31$ & $3.76 \pm 4.05$ & $-0.59 \pm 0.78$ \\
\hline
\end{tabular}

subjects had to resist the movement, we calculated the contribution as $-102.90 \pm 189.83$ percent. We compared these results with the approach using a measured reference support torque to calculate $C_{\text {user }}^{\prime}$. Mean difference between the two were $-1.43 \pm 1.26$ percent for the passive condition, $-2.92 \pm 8.10$ percent for the active condition, $-4.16 \pm 3.60$ percent for the half active condition, and $10.06 \pm 21.25$ percent for the resistive condition.

For patients with neurological problems, the estimated contribution during the passive arm elevation movement was $1.53 \pm 0.45$ percent. When instructed to move as actively as possible (active condition), we calculated a mean contribution of $51.74 \pm 44.20$ percent. P6 was unable to contribute to the movement. Instead, his voluntary effort led to a resistance to the movement. During the resistive condition, the estimated contribution was $-119.5 \pm 204.8$ percent. Comparing our approach with the approach using a measured reference torque showed a difference of $-1.54 \pm 0.53$ percent for the passive condition, $1.43 \pm 4.72$ percent for the active condition, and $2.43 \pm 16.01$ percent for the resistive condition.
Figure 6 shows boxplots of the results for the arm elevation movement for all participants.

\section{Horizontal Shoulder Rotation}

The contribution during the horizontal shoulder rotation was more difficult to estimate because the torques required to move the arms are relatively small since this joint is not affected by the gravity of the ARMin III or human arm. We excluded S4 because the arm model could not be identified. For the passive movement, we calculated a mean user contribution of $-0.02 \pm 2.03$ percent for nondisabled subjects. During the active condition, $C_{\text {user }}$ was $102.53 \pm 8.27$ percent. The mean contribution when the nondisabled subjects completed the half active condition was $21.49 \pm 48.28$ percent. Since torques that users can produce in this joint are much higher than the torques needed to move the arm, the instruction to "resist" led to very high negative values of 1,487.25 $\pm 1,367.80$ percent for $C_{u s e r}$ Comparing the calculated $C_{\text {user }}$ with the method where the measured reference torque is used showed a difference of $0.02 \pm$ 2.17 percent for the passive condition, $3.18 \pm 14.16$ percent 


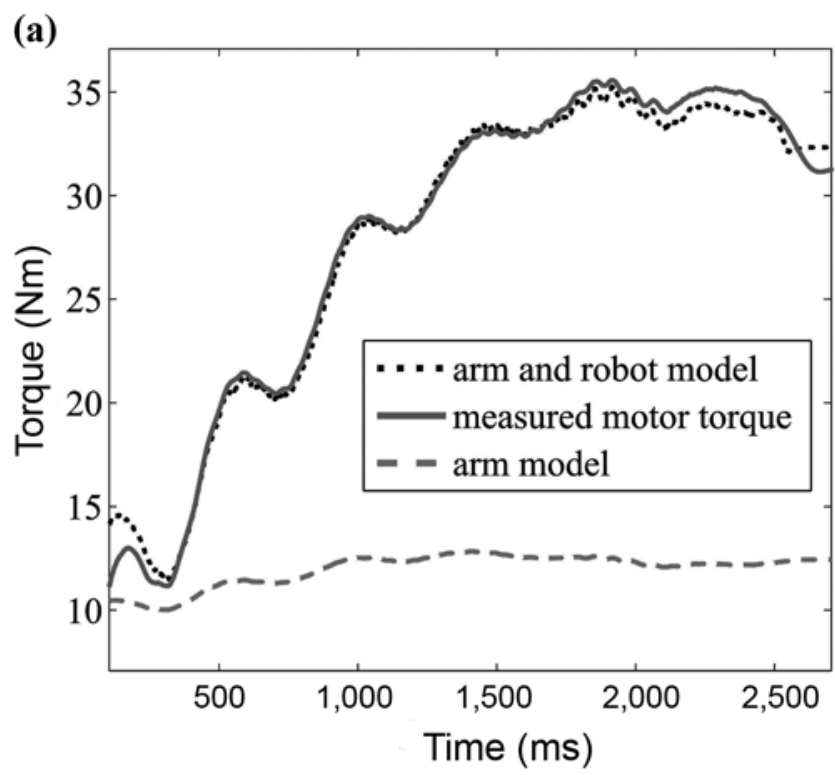

(c)



(b)

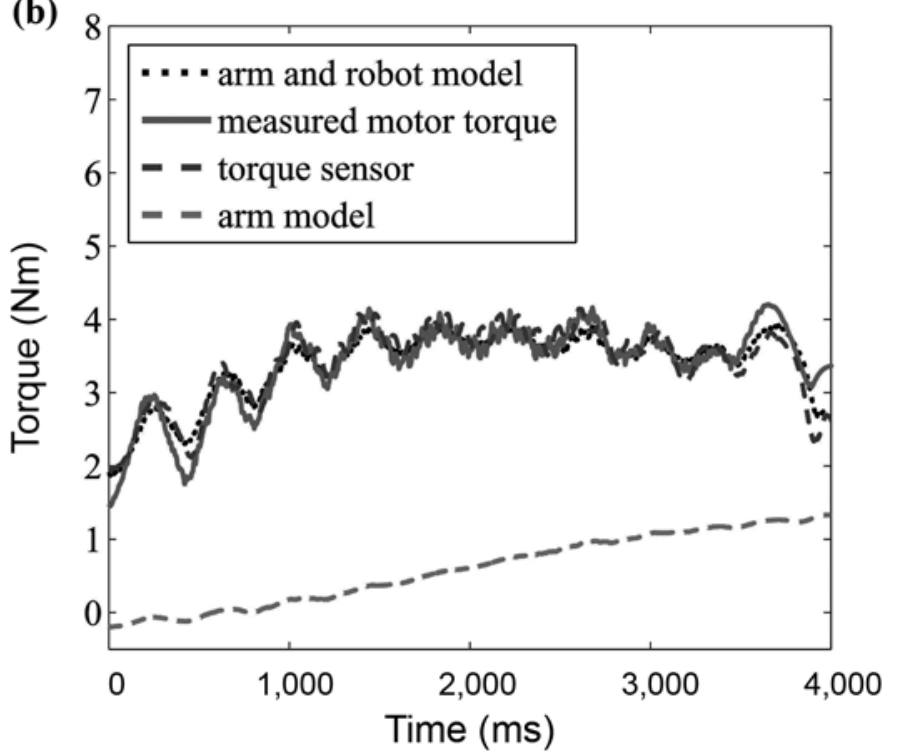

(d)

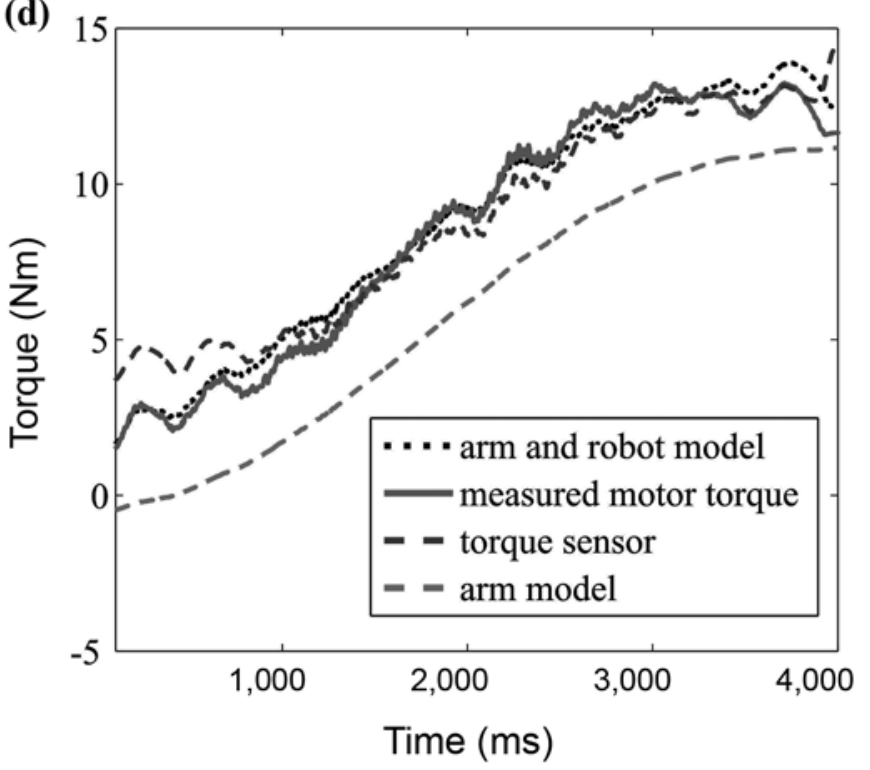

Figure 5.

Examples of measured and predicted model torque for passive arm movements. Gray dashed line represents identified arm model for subject. Additionally, we analyzed output of torque sensor for horizontal shoulder movement. (a) Passive arm elevation of nondisabled subject 5. (b) Passive horizontal shoulder movement of nondisabled shoulder 11. (c) Passive arm elevation of patient with neurological problem 2. (d) Passive horizontal shoulder movement of patient with neurological problem 6.

for the half active condition, $-2.53 \pm 9.14$ percent for the active condition, and $28.15 \pm 24.52$ percent for the resistive condition.

For patients with neurological problems, we calculated a contribution of $-1.3 \pm 3.6$ percent during the passive condition trials. The mean $C_{\text {user }}$ during the active condition was $41.9 \pm 49.6$ percent. Instructing patients to resist the movement led to a value of $-614.8 \pm 688.1$ percent. We excluded P1 from the results because we could not identify the arm model. Comparing our approach with the one without the human arm model led to a difference of $1.30 \pm 6.58$ percent for the passive condition, 
(a)

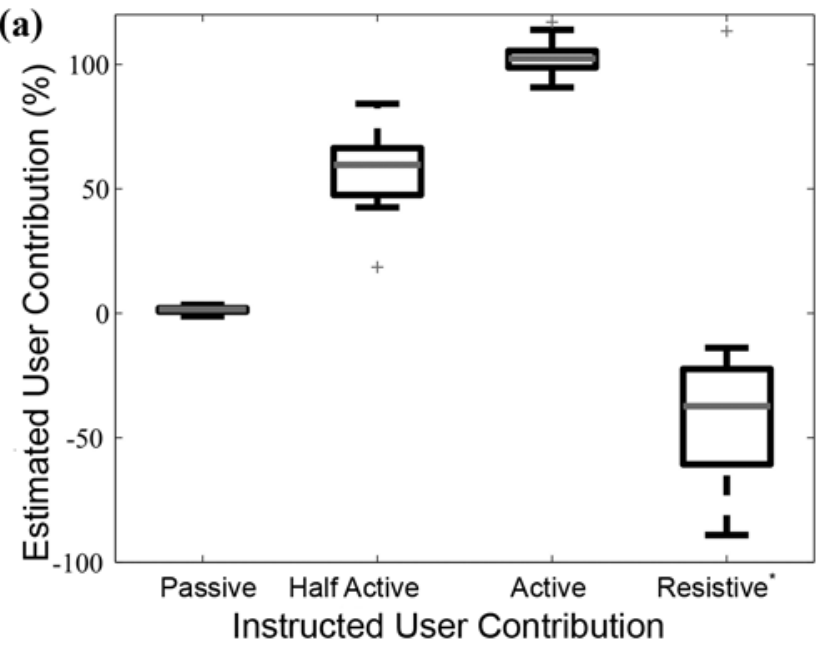

(c)

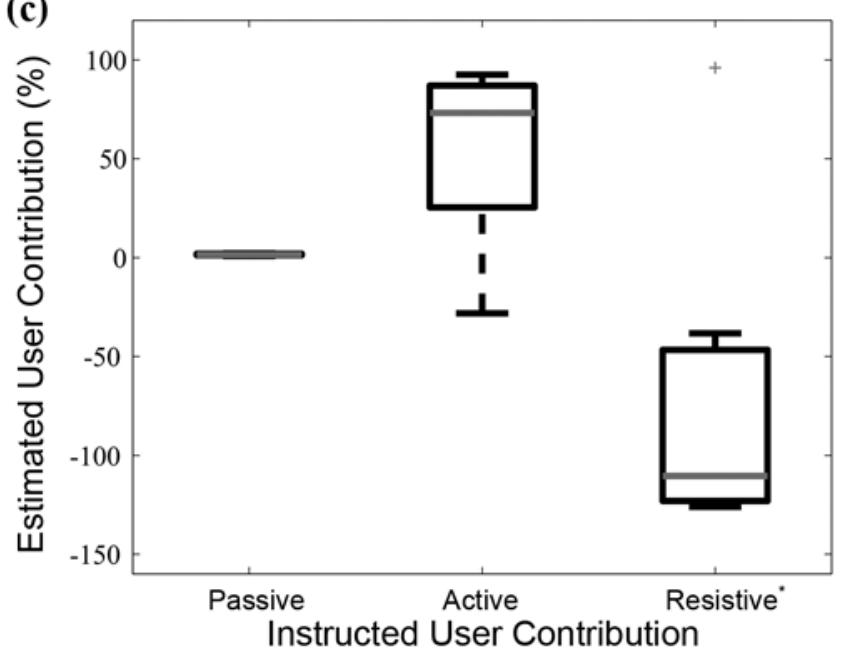

(b)

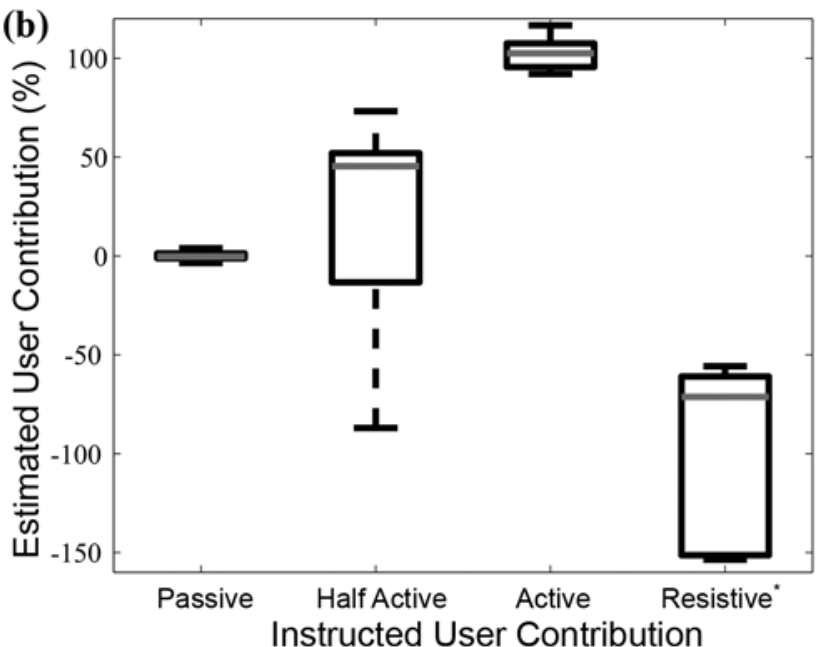

(d)



Figure 6.

Boxplot of estimated user contribution under given instructions. Resistive conditions indicated with * have been scaled to better fit into plot. (a) Arm elevation of nondisabled subjects. (b) Horizontal shoulder movement of nondisabled subjects. (c) Arm elevation of patients with neurological problems. (d) Horizontal shoulder movement of patients with neurological problems. $+=$ outliers of statistical analysis.

$-1.60 \pm 10.34$ percent for the active condition, and $7.69 \pm$ 130.20 percent for the resistive condition.

Figure 6 shows boxplots of the results for the horizontal shoulder rotation movement for all participants.

\section{DISCUSSION}

\section{Inverse Dynamic Robot Model}

For the proposed method to estimate the user's contribution, the dynamic model of the ARMin III is required. We calculated the model from $\mathrm{CAD}$ data and extended it with models of motor gear friction, the spring to counterbalance the weight of the robot, and the influence of its bending cables. Comparing the model-generated torques with measurements showed satisfying results for a rehabilitation robot. Adding the influence of the bending electronic cables on the exoskeleton visibly improved the model's accuracy (Figure 3).

The prediction accuracy of the model is quite good for nonzero velocities. Since static friction is not modeled, the prediction errors for joint velocities near zero are bigger (see Figure 5 at the beginning or end of the movement). The static friction parameters could also be 
extracted from the friction measurements and added to the model, but the nonlinearity of static friction makes it difficult to validate or use the model without direct measurement of the interaction forces with force or torque sensors [22].

\section{Inverse Dynamic Passive Human Arm Model}

We extended the rigid body model of the passive human arm consisting of inertia and gravitational effects with data obtained from measurements. In preliminary experiments, we observed an influence of joint position on the torque needed to move the passive arm. This position dependency is often explained as joint or muscle stiffness. Our identified passive arm stiffness is similar to values found in the literature [28]; also, the trajectories of gravity and inertia have a similar shape and amplitude as found in a previous study [29]. With the proposed simplified passive human arm model, the torques required to move a single joint could be estimated for the tested movement. The model for the horizontal shoulder actuation could not be identified for two nondisabled subjects because the three passive movements were too different. Possible reasons include trunk movements that changed the stiffness of the arm or difficulties in acting passively.

The developed arm model is not capable of capturing impairment characteristics of patients with stroke and should therefore be modified for further experiments. Effects due to muscle activation should be integrated. For instance, many patients with stroke show pathological synergies where voluntary torque in one joint evokes a torque in another joint; e.g., voluntarily abducting the arm leads to involuntary elbow flexion [30-31]. Arm measurements in other studies showed that the velocity of the joint has additional influence on the joint stiffness. Furthermore, a relationship between applied torque and stiffness was observed [32-33]. These effects might be considered for further extensions of the dynamic human arm model. Additionally, the ARMin III could be used to assess the exact weight of the upper arm and forearm of the individual user to optimally set up the gravity and inertia parameters of the model and thus reduce the total uncertainty of the method.

\section{User Contribution Estimation}

With the increasing intelligence of user-cooperative controllers, it is important to estimate the user contribution during robot-assisted therapy and feed back this information to the user and the therapist. Adapting the amount of assistance to the user's performance guarantees that he or she can complete the task, but carries the risk that he or she will decrease voluntary effort since the human motor system tends to optimize effort [34]. The proposed method to estimate the user's contribution can be used to observe and control effort during robotassisted therapy. Presenting the contribution in terms of percentage of the individual dynamic passive arm model allows a simple and comprehensive visualization of this contribution. The estimation of the user's contribution during passive movements is very accurate for patients with neurological problems and nondisabled subjects. This was expected since the passive human arm model is optimized with the measurements of the passive arm movements. However, the fact that the optimization was possible and the small SD between the trials show that the parameters of the model are able to replicate the required torque to move a passive arm.

We instructed nondisabled subjects to limit their effort in the half active condition to 50 percent. The mean estimated contribution was close to this instruction for the arm elevation movement. The large SD can be explained by the users' difficulty in generating exact levels of effort.

The result of the nondisabled subjects' half active condition and the patients' results trying to move during the active condition can be used to validate the method. In both cases, we could show that our model-based estimation of contribution is close to the common performance measure where a reference torque is obtained from a passive calibration movement and used to normalize the applied support torque. The estimated contribution for the arm elevation and horizontal shoulder movements during the active condition was similar for the individual patient with a neurological problem but did not correlate with FM score. Comparing only the proximal part of the FM score with the estimated activity improved the correlation, but more patients with neurological problems need to be evaluated to draw further conclusions (Appendix, available online only).

In the resistive condition, the instruction was not to produce an exact level of resistance because this would be very difficult for the user to achieve. Nevertheless, the results show that the resistive condition can be clearly distinguished from the other conditions. The high values of resistance for the horizontal shoulder rotation can be explained with the small mean arm torque required to perform that movement (dependent on the arm between 
0.7-11.0 Nm) and that some participants almost stopped the ARMin III during the movement, resulting in the maximal possible motor support torque of $59.5 \mathrm{Nm}$. Instead of acting against the movement, S4, P2, and P7 tried to move faster than the reference so the controller had to push them back. This resulted in a contribution value higher than 100 percent, which means they put more effort into the movement than required.

Since the current calculation of user contribution averages the torques over one movement, inertia effects could be neglected in the dynamic models because acceleration and deceleration torques cancel each other out.

\section{CONCLUSIONS}

This article presents and evaluates a method to estimate the user's contribution during robot-assisted therapy. The metric is based on inverse dynamic models of the robot and the passive human arm and was compared with a common performance measure where the mean reference support torque is calibrated for a particular movement. Evaluation with 12 nondisabled subjects and 7 patients with neurological problems showed that our method can calculate user effort based on recorded trajectories and motor torques similar to the common metric. Using a model-based approach has the advantage that it can be used for different movements once the model is identified. However, more effort is required to assess the dynamics of the human arm to build a more sophisticated arm model. Estimating and displaying the contribution of the user in a comprehensive way has the potential to increase active participation, which would be beneficial for the therapy outcome.

\section{ACKNOWLEDGMENTS}

\author{
Author Contributions: \\ Study concept and design: M. Guidali, U. Keller. \\ Acquisition of data: M. Guidali, U. Keller. \\ Analysis and interpretation of data: M. Guidali, U. Keller, T. Nef. \\ Critical revision of manuscript for important intellectual content: \\ M. Guidali, U. Keller, V. Klamroth-Marganska, T. Nef, R. Riener. \\ Study supervision: R. Riener.
}

Financial Disclosures: The authors have declared that no competing interests exist.

Funding/Support: This material was based on work supported by the Swiss National Science Foundation through the National Center of
Competence in Research Robotics and the Robot-Assisted Neurorehabilitation of the Arm (grant 325200-1260621).

Additional Contributions: The authors would like to thank the nondisabled subjects and especially the patients who participated in this study. We would also like to thank the therapists, Anja Kollmar and Sabine Schölch, for their help. Dr. Guidali is now with V-ZUG Ltd (Zug, Switzerland).

Institutional Review: All participants received written and verbal information about the study from the experimenter and gave written informed consent. The study protocol met the institutional guidelines and was approved by the institutional committees (Swissmedic and Cantonal Ethics Committee, Zurich, Switzerland).

Participant Follow-up: The authors do not plan to inform participants of the publication of this study. However, participants have been encouraged to check the institute Web site for updated publications.

\section{REFERENCES}

1. Nelles G, Jentzen W, Jueptner M, Müller S, Diener HC. Arm training induced brain plasticity in stroke studied with serial position emission tomography. Neuroimage. 2001; 13(6 Pt 1):1146-54. [PMID:11352620] http://dx.doi.org/10.1006/nimg.2001.0757

2. Lotze M, Braun C, Birbaumer N, Anders S, Cohen LG. Motor learning elicited by voluntary drive. Brain. 2003; 126(Pt 4):866-72. [PMID:12615644]

http://dx.doi.org/10.1093/brain/awg079

3. Hogan N, Krebs HI, Rohrer B, Palazzolo JJ, Dipietro L, Fasoli SE, Stein J, Hughes R, Frontera WR, Lynch D, Volpe BT. Motions or muscles? Some behavioral factors underlying robotic assistance of motor recovery. J Rehabil Res Dev. 2006;43(5):605-18. [PMID:17123202] http://dx.doi.org/10.1682/JRRD.2005.06.0103

4. Riener R, Lünenburger L, Jezernik S, Anderschitz M, Colombo G, Dietz V. Patient-cooperative strategies for robot-aided treadmill training: first experimental results. IEEE Trans Neural Syst Rehabil Eng. 2005;13(3):380-94. [PMID:16200761] http://dx.doi.org/10.1109/TNSRE.2005.848628

5. Marchal-Crespo L, Reinkensmeyer DJ. Review of control strategies for robotic movement training after neurologic injury. J Neuroeng Rehabil. 2009;6:20. [PMID:19531254] http://dx.doi.org/10.1186/1743-0003-6-20

6. Wolbrecht ET, Chan V, Reinkensmeyer DJ, Bobrow JE. Optimizing compliant, model-based robotic assistance to promote neurorehabilitation. IEEE Trans Neural Syst Rehabil Eng. 2008;16(3):286-97. [PMID:18586608] http://dx.doi.org/10.1109/TNSRE.2008.918389

7. Montagner A, Frisoli A, Borelli L, Procopio C, Bergamasco M, Carboncini MC, Rossi B. A pilot clinical study on robotic assisted rehabilitation in VR with an arm exoskeleton device. Proceedings of the 2007 IEEE International 
Conference on Virtual Rehabilitation; 2007 Sep 27-29; Venice, Italy. p. 57-64.

8. Loureiro RC, Harwin WS. Reach \& grasp therapy: Design and control of a 9-DOF robotic neuro-rehabilitation system. Proceedings of the IEEE 10th International Conference on Rehabilitation Robotics; 2007 Jun 13-15; Noordwijk aan Zee, the Netherlands. p. 757-63.

9. Reinkensmeyer DJ, Kahn LE, Averbuch M, McKennaCole A, Schmit BD, Rymer WZ. Understanding and treating arm movement impairment after chronic brain injury: progress with the ARM guide. J Rehabil Res Dev. 2000; 37(6):653-62. [PMID:11321001]

10. Colombo R, Pisano F, Micera S, Mazzone A, Delconte C, Carrozza MC, Dario P, Minuco G. Robotic techniques for upper limb evaluation and rehabilitation of stroke patients. IEEE Trans Neural Syst Rehabil Eng. 2005;13(3):311-24. [PMID:16200755] http://dx.doi.org/10.1109/TNSRE.2005.848352

11. Krebs HI, Palazzolo JJ, Dipietro L, Ferraro M, Krol J, Rannekleiv K, Volpe BT, Hogan N. Rehabilitation robotics: Performance-based progressive robot-assisted therapy. Auton Robots. 2003;15(1):7-20. http://dx.doi.org/10.1023/A:1024494031121

12. Lünenburger L, Colombo G, Riener R. Biofeedback for robotic gait rehabilitation. J Neuroeng Rehabil. 2007;4(1):1. [PMID:17244363] http://dx.doi.org/10.1186/1743-0003-4-1

13. Balasubramanian S, Wei R, Herman R, He J. Robot-measured performance metrics in stroke rehabilitation. Proceedings of the 2009 IEEE International Conference on Complex Medical Engineering; 2009 Apr 9-11; Tempe, AZ. p. 1-6.

14. Ziherl J, Novak D, Olenšek A, Mihelj M, Munih M. Evaluation of upper extremity robot-assistances in subacute and chronic stroke subjects. J Neuroeng Rehabil. 2010;7:52. [PMID:20955566] http://dx.doi.org/10.1186/1743-0003-7-52

15. Sciavicco L, Siciliano B. Modelling and control of robot manipulators. London (England): Springer; 2000.

16. Featherstone R, Orin D. Robot dynamics: Equations and algorithms. Proceedings of the 2000 IEEE International Conference on Robotics and Automation; 2000 Apr 24-28; San Francsico, CA. p. 826-34.

17. Atkeson CG. Learning arm kinematics and dynamics. Annu Rev Neurosci. 1989;12(1):157-83. [PMID:2648948] http://dx.doi.org/10.1146/annurev.ne.12.030189.001105

18. Nef T, Guidali M, Riener R. ARMin III - arm therapy exoskeleton with an ergonomic shoulder actuation. Appl Bionics Biomech. 2009;6(2):127-42. http://dx.doi.org/10.1080/11762320902840179

19. Guidali M, Duschau-Wicke A, Broggi S, KlamrothMarganska V, Nef T, Riener R. A robotic system to train activities of daily living in a virtual environment. Med Biol
Eng Comput. 2011;49(10):1213-23. [PMID:21796422]

http://dx.doi.org/10.1007/s11517-011-0809-0

20. Piras G, Cleghorn WL, Mills JK. Dynamic finite-element analysis of a planar high-speed, high-precision parallel manipulator with flexible links. Mech Mach Theory. 2005; 40(7):849-62.

http://dx.doi.org/10.1016/j.mechmachtheory.2004.12.007

21. Nef T, Mihelj M, Riener R. ARMin: a robot for patientcooperative arm therapy. Med Biol Eng Comput. 2007;45(9):887-900. [PMID:17674069] http://dx.doi.org/10.1007/s11517-007-0226-6

22. Nef T, Lum P. Improving backdrivability in geared rehabilitation robots. Med Biol Eng Comput. 2009;47(4): 441-47. [PMID:19184156] http://dx.doi.org/10.1007/s11517-009-0437-0

23. Olsson H, Åström KJ, Canudas de Wit C, Gäfvert M, Lischinsky P. Friction models and friction compensation. Eur J Control. 1998;4(3):176-95.

24. Winter DA. Biomechanics and motor control of human movement. New York (NY): Wiley; 1990.

25. Dolan JM, Friedman MB, Nagurka ML. Dynamic and loaded impedance components in maintenance of human posture. IEEE Trans Syst Man Cybern. 1993;23(3):698-709. http://dx.doi.org/10.1109/21.256543

26. Cavallaro EE, Rosen J, Perry JC, Burns S. Real-time myoprocessors for a neural controlled powered exoskeleton arm. IEEE Trans Biomed Eng. 2006;53(11):2387-96. [PMID:17073345] http://dx.doi.org/10.1109/TBME.2006.880883

27. Hughes RA, Cornblath DR. Guillain-Barré syndrome. Lancet. 2005;366(9497):1653-66. [PMID:16271648] http://dx.doi.org/10.1016/S0140-6736(05)67665-9

28. Bennett DJ, Hollerbach JM, Xu Y, Hunter IW. Time-varying stiffness of human elbow joint during cyclic voluntary movement. Exp Brain Res. 1992;88(2):433-42. [PMID:1577114] http://dx.doi.org/10.1007/BF02259118

29. Kodek T, Munih M. An analysis of static and dynamic joint torques in elbow flexion-extension movements. Simul Model Pract Theory. 2003;11(3-4):297-311. http://dx.doi.org/10.1016/S1569-190X(03)00063-7

30. Brunnstrom S. Movement therapy in hemiplegia. New York (NY): Harper and Row; 1970.

31. Dewald JP, Beer RF. Abnormal joint torque patterns in the paretic upper limb of subjects with hemiparesis. Muscle Nerve. 2001;24(2):273-83. [PMID:11180211]

32. Burdet E, Osu R, Franklin DW, Yoshioka T, Milner TE, Kawato M. A method for measuring endpoint stiffness during multi-joint arm movements. J Biomech. 2000;33(12): 1705-9. [PMID:11006397]

33. Perreault EJ, Kirsch RF, Crago PE. Effects of voluntary force generation on the elastic components of endpoint 
stiffness. Exp Brain Res. 2001;141(3):312-23.

[PMID:11715075]

http://dx.doi.org/10.1007/s002210100880

34. Todorov E. Optimality principles in sensorimotor control.

Nat Neurosci. 2004;7(9):907-15. [PMID:15332089]

http://dx.doi.org/10.1038/nn1309

Submitted for publication September 21, 2011. Accepted in revised form July 18, 2012.
This article and any supplementary material should be cited as follows:

Guidali M, Keller U, Klamroth-Marganska V, Nef T, Riener R. Estimating the patient's contribution during robotassisted therapy. J Rehabil Res Dev. 2013;50(3):379-94. http://dx.doi.org/10.1682/JRRD.2011.09.0172

\begin{tabular}{|c|c|}
\hline $\begin{array}{l}\text { Nu suevessions scresene or. } \\
\sqrt{ } \text { iThenticate: }\end{array}$ & Crosstef НЕнЕ \\
\hline 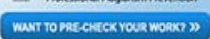 &  \\
\hline
\end{tabular}


